\title{
The role of NOI-domain containing proteins in plant immune signaling
}

Ahmed J Afzal ${ }^{1 *}$, Jin Hee Kim ${ }^{1}$ and David Mackey ${ }^{1,2}$

\begin{abstract}
Here we present an overview of our existing knowledge on the function of RIN4 as a regulator of plant defense and as a guardee of multiple plant R-proteins. Domain analysis of RIN4 reveals two NOI domains. The NOI domain was originally identified in a screen for nitrate induced genes. The domain is comprised of approximately 30 amino acids and contains 2 conserved motifs (PXFGXW and Y/FTXXF). The NOI gene family contains members exclusively from the plant lineage as far back as moss. In addition to the conserved NOI domain, members within the family also contain conserved C-terminal cysteine residue(s) which are sites for acylation and membrane tethering. Other than these two characteristic features, the sequence of the family of NOl-containing proteins is diverse and, with the exception of RIN4, their functions are not known. Recently published interactome data showing interactions between RIN4 and components of the exocyst complex prompt us to raise the hypothesis that RIN4 might be involved in defense associated vesicle trafficking.
\end{abstract}

\section{Introduction}

Microbes induce resistance responses in plants by activating two distinct branches of the plant immune system $[1,2]$. PTI (PAMP-triggered immunity) is activated upon recognition of pathogen associated molecular patterns (PAMPs), which are ubiquitous bacterial features such as epitopes within the bacterial flagellin protein $[3,4]$. PAMPs are recognized by pattern recognition receptors (PRR) and comprise the first layer of the plant defense response. Pathogens respond by delivering PTIsuppressing virulence factors, e.g. effector proteins injected from Gram-negative bacteria into the cytosol of plant cells through the needle-like Type III secretion system (TTSS). Furthering the arms race, plant-encoded resistance (R) proteins directly or indirectly recognize virulence effectors and activate effector-triggered immunity (ETI).

\section{R-protein activation - direct or indirect}

R-proteins are sometimes activated dependent on direct interaction with their cognate effector protein(s) [1]. For example, Y2H screens have showed that (1) the Pi-ta Rprotein of rice interacts with its cognate AvrPita effector

\footnotetext{
* Correspondence: afzal.5@osu.edu

${ }^{1}$ Department of Horticulture and Crop Science, The Ohio State University, Columbus, OH, USA

Full list of author information is available at the end of the article
}

from Magnaporthe grisea [5], (2) the L and M Rproteins from flax interact with their cognate AvrL567 and AvrM effectors from flax rust [6], and (3) the RRS1R R-protein of Arabidopsis thaliana interacts with its cognate PopP2 effector from Ralstonia solanacearum [7]. However, the lack of evidence of direct interaction for numerous other R-protein/cognate effector pairs led to formulation of the guard hypothesis, which states that R-proteins can indirectly recognize pathogen effectors by sensing perturbations they induce. The plant protein (or other type of molecule), of which a perturbed state is recognized by the R-protein, is known as the "guardee". Guardees can be either direct or indirect virulence targets of effectors or target decoys $[8,9]$.

Numerous studies over the last decade have lent support to the guard hypothesis. The Arabidopsis thaliana R-protein RPS5 is activated when PBS1 (serine threonine kinase), is cleaved by the Pseudomonas syringae cysteine protease effector protein, AvrPphB [10,11]. Another guardee protein, RIN4, is modified by at least four effectors from Pseudomonas syringae. HopF2, a T3E with ADP-ribosyltransferase activity targets RPM1-Interacting Protein 4 (RIN4) [12,13]. AvrB and AvrRpm1 induce phosphorylation of RIN4 [14-16] and AvrRpt2, a cysteine protease, cleaves RIN4 at two cleavage sites (RCS1 and RCS2) [17-19]. AvrB-, AvrRpm1-, and AvrRpt2-induced perturbations of RIN4 lead to the induction of ETI by at 
least two Arabidopsis thaliana R-proteins, RPM1 and RPS2 [14,15,20-23]. Whether HopF2-induced modification of RIN4 can also elicit R-protein mediated defense is not known.

R-proteins that "guard" RIN4 arose independently in a variety of plant species. RIN4 homologs regulate innate immunity in tomato [24] lettuce [25] and soybean [26]. Soybean contains 4 homologs of the RIN4 protein (GmRIN4a-GmRIN4d). Amino acid alignments show that GmRIN4a and GmRIN4b are 50\% identical to AtRIN4 whereas GmRIN4c and GmRIN4d are 46\% and 48\% identical to AtRIN4 respectively [26]. Resistance to AvrB and AvrRpm1 in soybean is mediated by the R-proteins RPG1-b and RPG1-r, respectively. Although functionally similar, RPG1-b and RPM1 are evolutionarily distinct [27]. Additional resistance genes specific to AvrRpm1, only one of which originates in the Rpg locus, have arisen in common bean [28]. In lettuce hybrids, necrotic lesions result from an allelic interaction between RIN4 (54\% identical to AtRIN4) and a second locus, with both loci contributing to quantitative resistance against a virulent race of Bremia lactucae and race specific resistance to an avirulent race of the same pathogen. It is tempting to speculate that the second locus that interacts with RIN4 is an R-gene. In any case, the independent evolution of R-proteins that guard RIN4 highlights the significance of RIN4 to plant defense.

Here we will describe in greater detail what is known about the function of RIN4 as a regulator of plant defense and as a guardee of multiple plant R-proteins.

\section{Sequence / structure analysis of RIN4 protein}

A lack of discernible structural features in RIN4 makes prediction of function difficult. Although domain analysis of RIN4 reveals two NOI domains (pfam05627), little is known about their role in plant defense. Proteins containing NOI domains were initially identified in a screen for nitrate-induced genes (NOI stands for $\mathrm{NO}_{3}$ Induced). No link between NOI proteins and nitrogen metabolism has been established. The family of proteins containing NOI domains contains members exclusively from the plant lineage as far back as moss. In addition to the conserved NOI domain, family members also contain conserved C-terminal cysteine residue(s). Other than these two characteristic features, NOI-containing proteins share no apparent homology and, with the exception of RIN4, their functions are not known.

RIN4 has two NOI domains located at the N- and C-terminal ends of the protein (termed N-NOI and C-NOI, respectively). We previously conducted phylogenetic analysis to examine the relation of $\mathrm{N}-\mathrm{NOI}$ and C-NOI protein domains from RIN4 and its most closely related homologs from a variety of other monocots and dicots, as well as, moss. Even though the proteins came from significantly diverged plants, the N-NOI and $\mathrm{C}$-NOI domains grouped into separate clades, indicating that they have evolved independently from one another [29]. Consistent with this interpretation are observations that the N-NOI and C-NOI of RIN4 function distinctly; the C-NOI interacts with AvrB and is required for RPM1-mediated ETI [15,30].

There are two conserved motifs within the NOI domains. The first conserved motif (PXFGXW) is the RIN4-cleavage site (RCS) targeted by AvrRpt2 [18]. With the exception of three RIN4 homologs from Arabidopsis thaliana (At5G48500, At5G48657 and At3G07195), all examined homologs contain an RCS in the C-terminal NOI domain. Cleavage of RCS2 by AvrRpt2 is critical for the activation of RPS2 [19,20]. The second conserved motif is the sequence Y/FTXXF, which is highly conserved in the center of the NOI domain.

The type III effectors AvrRpm1 and AvrB induce phosphorylation of the conserved threonine within the Y/FTXXF motif of both the N-NOI and C-NOI of Arabidopsis thaliana RIN4. Notably, threonine phosphorylation within the C-NOI is key to the activation of ETI by RPM1 $[14,15]$. Since effectors target specifically the two conserved motifs within the NOI domains of RIN4, they likely also target these same motifs within other NOI-containing proteins. In fact, in addition to RIN4, several other NOI-containing proteins from Arabidopsis thaliana were shown to be cleaved by AvrRpt2 [18,31]. Thus, it is a reasonable hypothesis that the NOI-family of proteins control plant processes, most likely biotic defense, that bacterial pathogens aim to perturb. The conservation of the RCS and Y/FTXXF motifs targeted by T3Es within the NOI domains likely results from the evolutionarily more ancient function of these motifs as targets for cellular proteins. Indeed, the phosphorylation of Arabidopsis thaliana RIN4 is mediated by an Arabidopsis thaliana receptor like protein kinase (RIPK) and, in tomato and tobacco, an endogenous protease has been shown to cleave the RCS $[14,24]$.

For our current analysis we generated a phylogenetic tree composed of 51 RIN4 homologs, each containing two NOI domains, from monocots, dicots and moss. The tree was generated using the entirely automated plant Ensembl database (http://plants.ensembl.org). The alignment composite generated from 51 RIN4 homologs shows greater sequence conservation and reduced gaps in the C-NOI in comparison to the N-NOI (Figure 1). We also analyzed the position of the exon junctions relative to the full-length protein. Most of the analyzed NOI proteins contain 3 introns. The intron that precedes the C-NOI (Intron 2) is conserved in all but 3 (Os03g63160, OB02G25470 and OB08G27970) sequences (Figure 2), while the intron preceding the N-NOI is conserved in 41 of the 51 analyzed proteins. Collectively, these data 


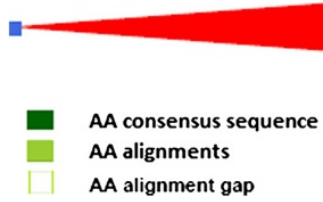

Figure 1 The protein alignment composite of $51 \mathrm{NOI}$ containing proteins from monocots, dicots and moss was generated using the Ensembl plant database (plants.ensembl.org/). The $51 \mathrm{NOI}$ containing proteins were selected on the basis of their sequence homology to AtRIN4. Green bars show areas of amino acid alignment while the white areas are gaps in the alignment. Dark green bars indicate the consensus alignment in the collapsed tree. The regions corresponding to the C- and N- NOI domains are shown by red bars. The highest level of conservation in sequence is seen in the C-NOI domain, followed by the N-NOI domain and the region representing the conserved C-terminal cysteines.

indicate that the C-NOI may be the ancestral version and most well conserved, while the N-NOI may have arisen through independent duplications that only sometimes retained the intron and are better able to diverge in amino acid sequence (Figure 2). The position of the third intron varies between monocots and dicots. This junction in most monocots and moss is one amino acid removed from the conserved cysteines, whereas in most dicots it immediately precedes the C-terminal cysteine residues (Figure 2). All analyzed RIN4 homologs from moss have additional introns between the two conserved introns. The most parsimonious explanation for exon evolution in angiosperms is that the additional junctions were lost after the split between moss and vascular plants. Alternatively, it is possible that the extra introns were lacking in the moss-vascular plant common ancestor and were acquired after divergence of moss.

We also generated alignments of 13 RIN4-like Arabidopsis proteins, selected on the basis of the plantspecific NOI domains and the conserved $\mathrm{C}$ terminal cysteine residues (Additional file 1: Figure S1). C-terminal sequences are important for sub-cellular localization of RIN4. With few exceptions, the NOI-containing RIN4 homologs (Figure 1), as well as other NOI containing proteins from Arabidopsis thaliana (Additional file 1: Figure S1), contain one to three cysteines within the Cterminal twelve amino acids that, except for in moss RIN4, are closely flanked by one or more aromatic residues [29]. In Arabidopsis thaliana RIN4, the C-terminal cysteines and the phenylalanine residues that follow are required for acylation and plasma membrane attachment [32]. Given the conservation of these residues at their $\mathrm{C}$-termini, acylation and membrane association is likely a common feature of NOI-containing proteins and may relate to their function. Consistent with this prediction, the C-terminal cysteines of RIN4 are required for supporting the function of RPM1 and negatively regulating the function of RPS2 (Luis da Cunha and D.M., unpublished data and [20]). Also, RIN4 derivatives lacking the C-terminal cysteines are hyperactive suppressors of PTI [29]. Thus, membrane tethering of RIN4 affects, both positively and negatively, its defense regulating functions in Arabidopsis thaliana. In Arabidopsis other than RIN4 only AT3G07195 and AT5G48657 contain both N- and C- terminal NOI domains. 3 of the examined NOI containing proteins (At5G48657, At5G48500 and At3G07195) lack the predictive AvrRpt2 cleavage site in the C-NOI domain. However these 3 proteins contain the conserved AvrRpt2 cleavage site (RCS motif) within the first 20 residues of the $\mathrm{N}$-terminus (Additional file 1: Figure S1). On the basis of the conservation of the AvrRpt2 cleavage site in the NOI-family of proteins, we can speculate that bacterial effectors aim to perturb a process important for biotic defense. Additionally, the conservation of the RCS motif targeted by T3Es within the NOI may allow NOIcontaining proteins to function as decoys [9].

\section{Role of NOI domains in suppression of PTI}

RIN4 was previously shown to negatively regulate PTI [33]. Over-expression of RIN4 suppressed PTI and PTI responses were enhanced in the absence of RIN4. To measure the relative contribution of the $\mathrm{N}$ - and $\mathrm{C}$-NOI domains in PTI regulation, the ability of over-expressed RIN4 derivatives to suppress flg22-induced callose deposition (flg22 is a PAMP from the bacterial flagellin protein) and enhance the growth of TTSS-deficient bacteria was determined [29]. A derivative of RIN4 lacking both NOI domains was unable to suppress PTI while derivatives lacking either NOI alone maintained the ability to suppress PTI. It therefore seems that the evolutionarily distinct $\mathrm{N}-\mathrm{NOI}$ and $\mathrm{C}-\mathrm{NOI}$ can each contribute to PTI suppression by over-expressed RIN4. However, this data does not exclude the possibility that distinct functions of each NOI domain would be detected under different experimental conditions or with different readouts for PTI.

AvrRpm1, AvrB, and AvrRpt2 can each perturb RIN4 and inhibit PTI [33,34]. Thus, it has been speculated that targeting of RIN4 contributes to the virulence function of these T3Es. However, in the case of AvrRpt2, it seemed counter-intuitive that this effector would cleave a negative regulator of PTI. A number of potential explanations have been provided for this observation. First, one or more NOI-containing proteins targeted by 

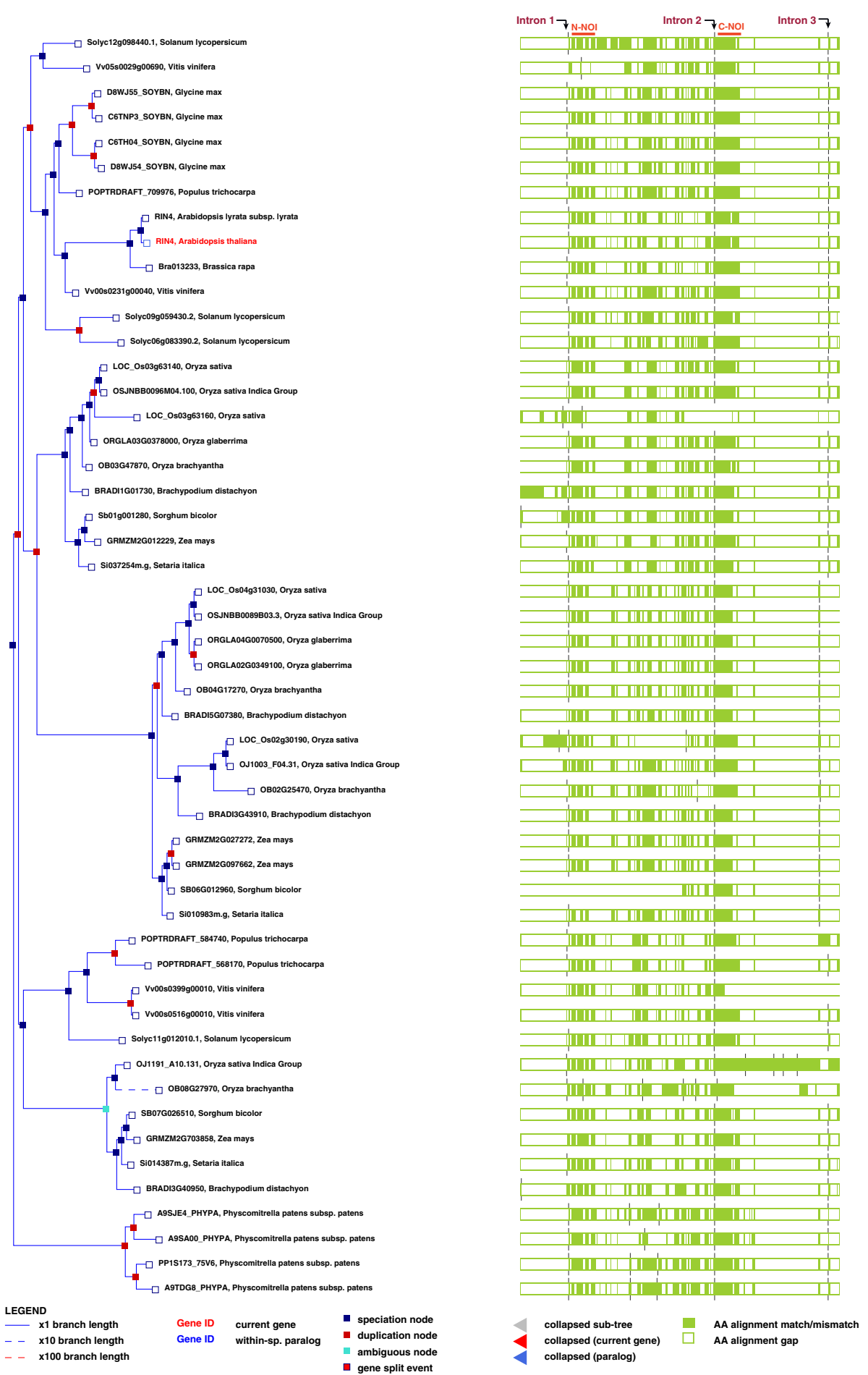

Figure 2 (Left panel) A Phylogenetic tree of 51 NOl containing proteins from monocots, dicots and moss was generated using the Ensembl plant database (plants.ensembl.org/). The $51 \mathrm{NOI}$ containing proteins were selected on the basis of their sequence homology to AtRIN4. Red nodes in the phylogenetic tree represent duplication events whereas the blue nodes are speciation events. (Right panel) Multiple alignments of the $51 \mathrm{NOI}$ containing proteins shown on the left were generated using MUSCLE. The light green bars correspond to amino acids within the protein sequence, whereas the white spaces represent alignment gaps. The 3 intron junctions are shown as black lines (labeled) that run perpendicular to the alignment trace. The intron junction preceding the N-NOI is labeled as intron 1 whereas the intron junction preceding the C-NOI is labeled as intron 2. The position of the third intron varies between monocots and dicots. This junction in most monocots and moss is one amino acid removed from the conserved cysteines, whereas in most dicots it immediately precedes the C-terminal cysteine residues. The regions corresponding to the N-NOI and C-NOI are shown by red bars. 
AvrRpt2 [18] may be bonafide virulence target(s) that regulate PTI. Consistent with this hypothesis is the observation that AvrRt2 is able to promote virulence in plants lacking RIN4 [27] presumably by targeting other NOI contacting proteins. Indeed, a recent study showed that inducible expression of AvrRpt2 decreases the level of three NOI-containing proteins in plasma membrane preparations of Arabidopsis thaliana (NOI4, NOI6 and NOI7) [31]. In this scenario, RIN4 might only serve as a decoy rather than as a bonafide virulence target of AvrRpt2 [9]. A second possibility is that degradation of RIN4 disrupts the function of associated proteins. For example, RIN4 interacts in guard cells with autoinhibited $\mathrm{H}^{+}$ATPases (AHA proteins) that promote the opening of stomata [35]. Stomatal closure is a PTI response that restricts bacterial invasion from the leaf surface [36]. $\mathrm{H}^{+}$ATPase activity is reduced in rin4 mutant plants, which tends to keep stomata closed and thus enhances this PTI response. However, disrupting the function of AHA proteins by elimination of RIN4 would enhance defense, contrary to the virulence function of AvrRpt2. Thus, in this scenario, elimination of RIN4 by AvrRpt2 would need to disrupt the function of another protein(s) that promote, rather than suppress, plant defense. Putative RIN4-interacting proteins involved in vesicle secretion, discussed below, may fit these criteria. Our recent findings support a third possibility that cleavage of RIN4 by AvrRpt2 does not simply eliminate RIN4, but rather produces fragments that effectively suppress PTI [29]. Consistent with this third idea, we have shown that the Avrpt2-cleavage products remain detectable in planta after cleavage of RIN4 by bacterially delivered AvrRpt2. Furthermore, the non-membrane localized ACP2 (AvrRpt2 Cleavage Product 2) and the membrane localized ACP3 (AvrRpt2 Cleavage Product 3) fragments are hyperactive PTI suppressors. ACP2, which is the fragment bounded by RCS1 and RCS2, contains most of the N-NOI and ACP3, which is the fragment C-terminal to RCS2, contains most of the C-NOI. According to the decoy model, a decoy functions by mimicking the actual effector target and should have no affect on pathogen fitness when the cognate $R$ protein is not activated [9]. Hence our data support the hypothesis that RIN4 is a bonafide virulence target of AvrRpt2 and indicates that cleavage of RIN4 by AvrRpt2 may activate its ability to suppress PTI.

We hypothesize that other effectors target RIN4 to enhance its defense suppressing activity. In soybean, GmRIN4 proteins regulate basal defense and may be virulence targets of AvrB and AvrRpm1 [26,37,38]. AvrB and AvrRpm1 induce phosporylation of Arabidopsis thaliana RIN4 at the conserved threonines within the $\mathrm{N}-\mathrm{NOI}$ and C-NOI $[14,15]$. It is therefore tempting to speculate that NOI phosphorylation suppresses plant defense. The ability of AvrB and AvrRpm1 to suppress PTI responses in plants lacking RIN4 indicates that RIN4 is not their only virulence target [33,39]. Defense suppression may also be mediated through targeting of the conserved threonine in other defense-regulating NOI-containing proteins. The action of HopF2 can suppress plant defense in a manner that requires RIN4, perhaps via ADP-ribosylation of RIN4 [12,13]. Cellular proteins that target these motifs within the NOI, e.g. plant proteins that cleave RCS sites or phosphorylate the conserved threonine [14,24], may function as defense regulators. In this scenario, the effectors that suppress defense by modifying RIN4 and other NOI proteins have co-opted a cellular defense-regulatory process.

\section{Role of NOI domains in activation of ETI}

Our RIN4 structural model [29] predicts that the two NOIs interact extensively. This could help explain the observed intermolecular interactions between RIN4 homologs in soybean, which may be required for function of the Rpg1-b R-protein [26]. Structural changes and sub-cellular relocation brought about by T3E perturbations could activate R-proteins by promoting or disrupting intra- or inter-molecular NOI:NOI interactions and/or interactions of RIN4 with heterologous protein partners.

In Arabidopsis thaliana, RPM1 and RPS2 are predicted to be cytosolic, but localize to the plasma membrane $[21,35,40]$. RIN4 prevents ectopic activation of RPM1 and RPS2, perhaps via their interaction at the plasma membrane $[16,20-22,39]$. The C-NOI domain of RIN4 can complement AvrB and AvrRpm1 triggered RPM1 function whereas N-NOI is unable to do so. Both effectors associate with and induce phosphorylation of RIN4 although neither has known kinase activity [16]. Recently RIPK has been identified as the kinase that phosphorylates RIN4 at three residues (T21, S160 and T166) in both NOI domains [14]. Phospho-mimic substitutions at T166 in the C-NOI caused effectorindependent RPM1 activation. However, T166 is not essential for AvrRpm1 dependent HR in N. Benthamiana but is required for AvrB mediated RPM1 activation in Arabidopsis [15]. AvrRpm1- or AvrB-induced phosphorylation does not release RIN4 from the plasma membrane and leads to the activation of RPM1 at the plasma membrane [41]. Phosphorylated RIN4 may activate RPM1 by recruiting proteins required for R-protein mediated signaling or alleviate its negative regulation of RPM1. A residue analogous to T166 is conserved in all NOI containing proteins, hence this residue may function beyond the regulation of RPM1 [15].

The subcellular location of RPS2 activation is unknown. The ectopic activation of RPS2 in rin4 mutant plants differs genetically from AvrRpt2-induced activation of 
RPS2. RPS2-induced HR in response to AvrRpt2 is nonrace-specific disease resistance 1 (NDR1)-dependent [28] whereas the ectopic activation of RPS2 in plants lacking rin4 is NDR1-independent [39]. Interestingly, ACP3 of RIN4 has been shown to interact with plasma membrane localized NDR1 [42]. Thus, activation of RPS2 by AvrRpt2 may require signaling steps including NDR1:ACP3 interaction and a novel function for ACP2 that could include release from the plasma membrane and movement to a new sub-cellular location.

\section{Putative RIN4-interacting proteins}

To determine additional targets of RIN4 and other Arabidopsis thaliana NOI containing proteins, we searched the Arabidopsis Interactome Mapping database (AI-1) and the plant-pathogen immune network database (PPIN-1) [43,44]. PPIN-1 contains 3148 interactions, 843 Arabidopsis thaliana immune related proteins and 83 combined effectors from Pseudomonas syringae and Hyaloperonospora arabidopsidis whereas the AI-1 network contains 6205 interactions and 2,774 Arabidopsis thaliana proteins. We found 9 interacting partners for RIN4 using the PPIN-1 database (Table 1). The known RIN4 interactors RPM1 [16] and RPS2 [21] were missed in the screen, although for RPS2 it is not clear if the interaction is direct or indirect. Other known RIN4 interactors, AvrB and NDR1, were detected (Table 1). An uncharacterized CC-NB-LRR protein (AT1G12290) was also found to interact with RIN4, raising the possibility of RIN4 being guarded by additional R-proteins. From PPIN-1, we found interactors for 2 NOI containing proteins. We also found interactors for 2 of the 10 Arabidopsis thaliana NOI-containing proteins represented in AI-1. NOI6 and RIN4, each interact with proteins belonging to the Cys/His-rich and Exo70 protein families. Thus, interactions with these proteins likely are mediated through the NOI domain. The AI-1 and PPIN-1 databases contain only a subset of the entire immune repertoire, thus many NOI-R interactions were not detected. However, the existence of $15 \mathrm{NOI}$-containing proteins in Arabidopsis thaliana and of similarly large gene families in other sequenced plants indicates that they play generally important roles in plants, including perhaps plant defense, and therefore may be guarded by R-proteins.

The evolutionarily most ancient function of RIN4 may have been in the regulation of PTI, specifically limiting the detrimental effects of inappropriate defense signaling. As a defense regulator, it may have been a ripe target for bacterial effector proteins, and consequently for subsequent guarding by R-proteins. R-protein activation emanating from perturbation of RIN4 might involve cellular reprogramming that restores the signaling pathway originally targeted by T3Es. Supporting this idea are observations that numerous genes are required for both PTI- and ETI-signaling, e.g. phytoalexin deficient 4 (PAD4) and NDR1 [45] and that there is significant overlap in the immune responses, such as the expression of defense related genes and callose deposition, during PTI and ETI [46,47]. More generally, ETI may be envisioned as the protection/restoration and amplification of PTI responses. Thus, proteins that interact with RIN4 in a biologically significant manner might have roles in PTI and/or ETI.

\section{A putative role for RIN4 in regulating defense-associated vesicle trafficking}

An ADP ribosylation factor-guanine nucleotide exchange factor (ARF-GEF) protein HopM1 interacting protein 7

Table 1 Interactors of RIN4 and NOI containing proteins in the AI-1 and PPIN-1 databases

\begin{tabular}{|c|c|c|}
\hline Accession & Interactors (PPIN-1) & Interactors (Al-1) \\
\hline \multirow[t]{9}{*}{ RIN4 (AT3G25070) } & Unknown (AT3G01670) & \\
\hline & Cysteine/Histidine-rich (AT3G46810) & \\
\hline & EXO70E2 (AT5G61010) & \\
\hline & EXO70B1 (AT5G58430) & \\
\hline & NDR1 (AT3G20600) & \\
\hline & NB-LRR (AT1G12290) & \\
\hline & Cysteine/Histidine-rich (AT2G19650) & \\
\hline & bZIP transcription factor (AT3G51960) & \\
\hline & Pseudomonas syringae effector AvrB & \\
\hline NOI9 (AT5G48500) & Anaphase-promoting complex (AT3G48150) Unknown (AT4G01090) & Anaphase-promoting complex (AT3G48150) \\
\hline NOI3 (AT2G17660) & HSP81-3 (AT5G56010) & \\
\hline \multirow[t]{2}{*}{ N0I6 (AT5G64850) } & & Cysteine/Histidine-rich (AT4G01920) \\
\hline & & EXO70A1 (AT5G03540) \\
\hline
\end{tabular}

Targets of RIN4 and other Arabidopsis thaliana NOI containing proteins were determined using the Arabidopsis Interactome Mapping database (Al-1) and the plant-pathogen immune network database (PPIN-1). The PPIN-1 contains 3148 interactions, 843 Arabidopsis immune related proteins and 83 combined effectors from Pseudomonas syringae and Hyaloperonospora arabidopsidis whereas the Al-1 network contains 6205 interactions and 2,774 Arabidopsis proteins. From PPIN-1, we found interactors for $3 \mathrm{NOI}$ containing proteins whereas the Al-I database search resulted in interactors for 2 NOI-containing proteins. 
(AtMIN7/BIG5) is required for elicitation of both PTI and ETI in Arabidopsis thaliana. The protein is a vesicle traffic regulator in Arabidopsis thaliana which is destabilized by HopM1, a type III effector protein from $P$. syringae [48-50]. It is therefore likely that one mode of PTI suppression by bacterial effectors is mediated by an ability to interfere with the defense-associated vesicle trafficking pathway. Activation of AvrRpt2-induced ETI results in the stabilization of AtMIN7 in transgenic plants expressing HopMI, thereby reinforcing the PTI response [48]. AvrRpt2-mediated RPS2 activation also induces the activation of additional proteins involved in vesicular trafficking [31]. Thus, AvrRpt2-induced perturbation of RIN4 and the subsequent activation of RPS2 can be envisioned as a restoration of the vesicular trafficking network necessary for PTI. Our previous studies failed to show an effect of RIN4 over-expression on early signaling events during PTI [29]. Moreover, the PPIN-1 and AI-1 interactors for RIN4 and NOI6 include EXO70 proteins (Table 1). Interactions between RIN4 and a component of the exocyst complex, which regulates vesicle trafficking, prompts us to raise the hypothesis that RIN4 might be involved in defense-associated vesicle trafficking. RIN4 may guide or activate the polarized secretion of defense-related vesicles toward the bacterial infection site. Alternatively, EX070 complexes might recognize newly synthesized RIN4 proteins, driving RIN4 movement toward plasma membranes under pathogen attack.

The plant immune system uses secretory vesicles to transfer defense-related cargoes toward the plasma membrane at the site of pathogen invasion [51]. Several studies have suggested that the secretory system is one of the crucial factors in the battle between pathogen attack and host defense. Pathogen effectors inhibit host trafficking. The suite of $P$. syringe type III effectors preferentially repress a set of Arabidopsis thaliana genes encoding secreted proteins and disrupt expression of genes involved in the secretion processes $[52,53]$. $X$. campestris $p v$. vesicatoria effector protein XopJ inhibits apoplastic accumulation of a secreted version of GFP [54]. HopZ1a expressed by some strains of $P$. syringae disrupts the microtubule network and disrupts secretion [55]. On the other hand, host plants have adapted to increase capacity of the secretory system during induced defense. SA-dependent activation of non-expressor of pathogenesis related 1 (NPR1) regulates the induction of $P R$ genes, the products of which are secreted proteins, as well as a large set of genes encoding proteins involved in facilitating secretion [56]. Expression of exocyst components including EXO70B2, H1, H2, and $\mathrm{H} 7$ is responsive to treatment of elicitor proteins $[53,57,58]$. Mutations of Exo70B1 or Exo70H2 enhance susceptibility to fungal and bacterial infection [58].

\section{Conclusions}

In the last decade, significant progress has been made towards examining the role of RIN4 as a plant defense regulator. Interaction of RIN4 with the exocyst complex prompts us to raise the hypothesis that RIN4 might be involved in defense associated vesicle trafficking. Although the importance of vesicle-mediated transport in plant immunity is well established, knowledge of the contents of defense-associated vesicles is limited. Also, the regulatory mechanisms that connect recognition of pathogen to enhanced vesicle transport and the molecular mechanisms by which vesicle trafficking is polarized toward the site of infection need better resolved. The role of RIN4 in one or more of these processes remains to be determined.

\section{Additional file}

Additional file 1: Figure S1. ClustalW alignment of RIN4 and 13 Arabidopsis NOI containing proteins (AT5G48657, AT5G48500, AT3G07195, AT5G19473, AT5G09960, AT5G64850, AT5G55850, AT2G04410, AT5G63270, AT3G48450, AT5G40645, AT2G17660 and AT4G35655) was performed on the EBI server. The C-NOI domain is outlined in orange whereas the C-terminal cysteine residues are highlighted by the black box. The AvrRpt2 cleavage site (red arrow) lies within the consensus PxFGxW motif (red box). In addition to RIN4 only AT3G07195 and AT5G48657 contain both $\mathrm{N}$ - and C-NOI domains. 3 out of the 14 proteins (At5G48657, At5G48500 and At3G07195) lack the predictive AvrRpt2 cleavage site in the C-NOI domain. However the 3 proteins contain the conserved AvrRpt2 cleavage site within 20 amino acids of the $\mathrm{N}$-terminus. All the analyzed proteins contain 1-3 C-terminal cysteine residues required for palmitoylation and membrane attachment found in RIN4. The alignments were edited and visualized using Jalview.

\section{Abbreviations}

NOI: Nitrate induced; PAMP: Pathogen-associated molecular patters; ETI: Effector-triggered immunity; PTI: PAMP-triggered immunity; TTSS: Type III secretion system; RIN4: RPM1 interacting protein 4; RCS: RIN4 cleavage site; PPIN: Plant-pathogen immune network; NDR1: Non-race-specific disease resistance 1; ARF-GEF: ADP ribosylation factor-guanine nucleotide exchange factor; PAD4: Phytoalexin deficient 4; CC-NB-LRR: Coiled coil-nucleotide binding-leucine rich repeat; ACP: AvrRpt2-cleavage product; PR1: Pathogenesis related 1; NPR1: Non-expressor of pathogenesis related 1.

\section{Competing interests}

Authors declare that they have no competing interests.

\section{Authors' contributions}

DM and AJA conceived of the study. AJA carried out the NOI interactome analysis and generated the phylogenetic tree and protein alignments. DM, AJA and JHK wrote the manuscript. AJA and DM edited the final version. All authors read and approved the final manuscript.

\section{Acknowledgements}

We thank Jeff Dangl for insightful discussions. This work was funded by the National Science Foundation grant MCB-0718882, the Ohio Agricultural Research and Development Center of The Ohio State University, and the Korean RDA Next-Generation BioGreen Program (SSAC and PJ009088). Any opinions, findings, and conclusions or recommendations expressed in this material are those of the author(s) and do not necessarily reflect the views of the funding agencies. 


\section{Author details}

'Department of Horticulture and Crop Science, The Ohio State University, Columbus, OH, USA. ²Department of Molecular Genetics, The Ohio State University, Columbus, OH, USA

Received: 13 June 2012 Accepted: 26 March 2013

Published: 14 May 2013

\section{References}

1. Jones JD, Dangl JL: The plant immune system. Nature 2006, 444(7117):323-329.

2. Chisholm ST, Coaker G, Day B, Staskawicz BJ: Host-microbe interactions: shaping the evolution of the plant immune response. Cell 2006 , 124(4):803-814.

3. Boller $T$, He SY: Innate immunity in plants: an arms race between pattern recognition receptors in plants and effectors in microbial pathogens. Science 2009, 324(5928):742-744.

4. Cai R, Lewis J, Yan S, Liu H, Clarke CR, Campanile F, Almeida NF, Studholme DJ, Lindeberg M, Schneider D, et al: The plant pathogen Pseudomonas syringae pv. tomato is genetically monomorphic and under strong selection to evade tomato immunity. PLoS pathogens 2011, 7(8):e1002130.

5. Jia Y, McAdams SA, Bryan GT, Hershey HP, Valent B: Direct interaction of resistance gene and avirulence gene products confers rice blast resistance. EMBO J 2000, 19(15):4004-4014.

6. Dodds PN, Lawrence GJ, Catanzariti AM, Teh T, Wang Cl, Ayliffe MA, Kobe B, Ellis JG: Direct protein interaction underlies gene-for-gene specificity and coevolution of the flax resistance genes and flax rust avirulence genes. Proc Natl Acad Sci USA 2006, 103(23):8888-8893.

7. Deslandes L, Olivier J, Peeters N, Feng DX, Khounlotham M, Boucher C, Somssich I, Genin S, Marco Y: Physical interaction between RRS1-R, a protein conferring resistance to bacterial wilt, and PopP2, a type III effector targeted to the plant nucleus. Proc Natl Acad Sci USA 2003 , 100(13):8024-8029.

8. Van der Biezen EA, Jones JD: Plant disease-resistance proteins and the gene-for-gene concept. Trends Biochem Sci 1998, 23(12):454-456.

9. van der Hoorn RA, Kamoun S: From Guard to Decoy: a new model for perception of plant pathogen effectors. Plant Cell 2008, 20(8):2009-2017.

10. Shao F, Golstein C, Ade J, Stoutemyer M, Dixon JE, Innes RW: Cleavage of Arabidopsis PBS1 by a bacterial type III effector. Science 2003, 301(5637):1230-1233.

11. Ade J, DeYoung BJ, Golstein C, Innes RW: Indirect activation of a plant nucleotide binding site-leucine-rich repeat protein by a bacterial protease. Proc Natl Acad Sci USA 2007, 104(7):2531-2536.

12. Wilton M, Subramaniam R, Elmore J, Felsensteiner C, Coaker G, Desveaux D: The type III effector HopF2Pto targets Arabidopsis RIN4 protein to promote Pseudomonas syringae virulence. Proc Natl Acad Sci USA 2010, 107(5):2349-2354

13. Wang Y, Li J, Hou S, Wang X, Li Y, Ren D, Chen S, Tang X, Zhou JM: A Pseudomonas syringae ADP-ribosyltransferase inhibits Arabidopsis mitogen-activated protein kinase kinases. Plant Cell 2010, 22(6):2033-2044

14. Liu J, Elmore JM, Lin ZJ, Coaker G: A receptor-like cytoplasmic kinase phosphorylates the host target RIN4, leading to the activation of a plant innate immune receptor. Cell Host Microbe 2011, 9(2):137-146.

15. Chung EH, da Cunha L, Wu AJ, Gao Z, Cherkis K, Afzal AJ, Mackey D, Dangl $J$ L: Specific threonine phosphorylation of a host target by two unrelated type III effectors activates a host innate immune receptor in plants. Cell Host Microbe 2011, 9(2):125-136.

16. Mackey D, Holt BF 3rd, Wiig A, Dangl JL: RIN4 interacts with Pseudomonas syringae type III effector molecules and is required for RPM1-mediated resistance in Arabidopsis. Cell 2002, 108(6):743-754

17. Axtell MJ, Chisholm ST, Dahlbeck D, Staskawicz BJ: Genetic and molecular evidence that the Pseudomonas syringae type III effector protein AvrRpt2 is a cysteine protease. Mol Microbio/ 2003, 49(6):1537-1546.

18. Chisholm ST, Dahlbeck D, Krishnamurthy N, Day B, Sjolander K, Staskawicz $B J$ : Molecular characterization of proteolytic cleavage sites of the Pseudomonas syringae effector AvrRpt2. Proc Natl Acad Sci USA 2005, 102(6):2087-2092.

19. Kim HS, Desveaux D, Singer AU, Patel P, Sondek J, Dangl JL: The Pseudomonas syringae effector AvrRpt2 cleaves its C-terminally acylated target, RIN4, from Arabidopsis membranes to block RPM1 activation. Proc Natl Acad Sci USA 2005, 102(18):6496-6501.
20. Day B, Dahlbeck D, Huang J, Chisholm ST, Li D, Staskawicz BJ: Molecular basis for the RIN4 negative regulation of RPS2 disease resistance. Plant Cell 2005, 17(4):1292-1305.

21. Axtell MJ, Staskawicz BJ: Initiation of RPS2-specified disease resistance in Arabidopsis is coupled to the AvrRpt2-directed elimination of RIN4. Cell 2003, 112(3):369-377.

22. Mackey D, Belkhadir Y, Alonso JM, Ecker JR, Dangl JL: Arabidopsis RIN4 is a target of the type III virulence effector AvrRpt2 and modulates RPS2mediated resistance. Cell 2003, 112(3):379-389.

23. Kim MG, Geng X, Lee SY, Mackey D: The Pseudomonas syringae type III effector AvrRpm1 induces significant defenses by activating the Arabidopsis nucleotide-binding leucine-rich repeat protein RPS2. The Plant journal : for cell and molecular biology 2009, 57(4):645-653.

24. Luo Y, Caldwell KS, Wroblewski T, Wright ME, Michelmore RW: Proteolysis of a negative regulator of innate immunity is dependent on resistance genes in tomato and Nicotiana benthamiana and induced by multiple bacterial effectors. Plant Cell 2009, 21(8):2458-2472

25. Jeuken MJ, Zhang NW, McHale LK, Pelgrom K, den Boer E, Lindhout $P$, Michelmore RW, Visser RG, Niks RE: Rin4 causes hybrid necrosis and racespecific resistance in an interspecific lettuce hybrid. Plant Cell 2009, 21(10):3368-3378.

26. Selote D, Kachroo A: RPG1-B-derived resistance to AvrB-expressing Pseudomonas syringae requires RIN4-like proteins in soybean. Plant Physiol 2010, 153(3):1199-1211.

27. Lim MT, Kunkel BN: The Pseudomonas syringae type III effector AvrRpt2 promotes virulence independently of RIN4, a predicted virulence target in Arabidopsis thaliana. The Plant journal: for cell and molecular biology 2004, 40(5):790-798.

28. Century KS, Holub EB, Staskawicz BJ: NDR1, a locus of Arabidopsis thaliana that is required for disease resistance to both a bacterial and a fungal pathogen. Proc Natl Acad Sci USA 1995, 92(14):6597-6601.

29. Afzal AJ, da Cunha L, Mackey D: Separable fragments and membrane tethering of Arabidopsis RIN4 regulate its suppression of PAMPtriggered immunity. Plant Cell 2011, 23(10):3798-3811.

30. Desveaux D, Singer AU, Wu AJ, McNulty BC, Musselwhite L, Nimchuk Z, Sondek J, Dangl JL: Type III effector activation via nucleotide binding, phosphorylation, and host target interaction. PLoS Pathog 2007, 3(3):e48.

31. Elmore JM, Liu J, Smith B, Phinney B, Coaker G: Quantitative proteomics reveals dynamic changes in the plasma membrane during Arabidopsis immune signaling. Molecular \& cellular proteomics: MCP 2012, 11(4):M111 014555

32. Takemoto D, Jones DA: Membrane release and destabilization of Arabidopsis RIN4 following cleavage by Pseudomonas syringae AvrRpt2. Molecular plant-microbe interactions: MPMI 2005, 18(12):1258-1268.

33. Kim MG, da Cunha L, McFall AJ, Belkhadir Y, DebRoy S, Dangl JL, Mackey D: Two Pseudomonas syringae type III effectors inhibit RIN4-regulated basal defense in Arabidopsis. Cell 2005, 121(5):749-759.

34. Shang Y, Li X, Cui H, He P, Thilmony R, Chintamanani S, Zwiesler-Vollick J, Gopalan S, Tang X, Zhou JM: RAR1, a central player in plant immunity, is targeted by Pseudomonas syringae effector AvrB. Proc Natl Acad Sci USA 2006, 103(50):19200-19205.

35. Liu J, Elmore JM, Fuglsang AT, Palmgren MG, Staskawicz BJ, Coaker G: RIN4 functions with plasma membrane $\mathrm{H}+-$ ATPases to regulate stomatal apertures during pathogen attack. PLOS Biol 2009, 7(6):e1000139.

36. Melotto M, Underwood W, Koczan J, Nomura K, He SY: Plant stomata function in innate immunity against bacterial invasion. Cell 2006, 126(5):969-980.

37. Ashfield T, Ong LE, Nobuta K, Schneider CM, Innes RW: Convergent evolution of disease resistance gene specificity in two flowering plant families. Plant Cell 2004, 16(2):309-318.

38. Ong $L E$, Innes RW: AvrB mutants lose both virulence and avirulence activities on soybean and Arabidopsis. Mol Microbiol 2006, 60(4):951-962.

39. Belkhadir Y, Nimchuk Z, Hubert DA, Mackey D, Dangl JL: Arabidopsis RIN4 negatively regulates disease resistance mediated by RPS2 and RPM1 downstream or independent of the NDR1 signal modulator and is not required for the virulence functions of bacterial type III effectors AvrRpt2 or AvrRpm1. Plant Cell 2004, 16(10):2822-2835.

40. Boyes DC, Nam J, Dangl JL: The Arabidopsis thaliana RPM1 disease resistance gene product is a peripheral plasma membrane protein that is degraded coincident with the hypersensitive response. Proc Natl Acad Sci USA 1998, 95(26):15849-15854. 
41. Gao Z, Chung EH, Eitas TK, Dangl JL: Plant intracellular innate immune receptor Resistance to Pseudomonas syringae pv. maculicola 1 (RPM1) is activated at, and functions on, the plasma membrane. Proc Natl Acad Sci USA 2011, 108(18):7619-7624.

42. Day B, Dahlbeck D, Staskawicz BJ: NDR1 interaction with RIN4 mediates the differential activation of multiple disease resistance pathways in Arabidopsis. Plant Cell 2006, 18(10):2782-2791.

43. Mukhtar MS, Carvunis AR, Dreze M, Epple P, Steinbrenner J, Moore J, Tasan M, Galli M, Hao T, Nishimura MT, et al: Independently evolved virulence effectors converge onto hubs in a plant immune system network. Science 2011, 333(6042):596-601.

44. Dreze M: Evidence for network evolution in an Arabidopsis interactome map. Science 2011, 333(6042):601-607.

45. Fellbrich G, Romanski A, Varet A, Blume B, Brunner F, Engelhardt S, Felix G, Kemmerling B, Krzymowska M, Nurnberger T: NPP1, a Phytophthoraassociated trigger of plant defense in parsley and Arabidopsis. The Plant journal: for cell and molecular biology 2002, 32(3):375-390

46. Navarro L, Zipfel C, Rowland O, Keller I, Robatzek S, Boller T, Jones JD: The transcriptional innate immune response to flg22. Interplay and overlap with Avr gene-dependent defense responses and bacterial pathogenesis. Plant physiology 2004, 135(2):1113-1128.

47. Gao M, Wang X, Wang D, Xu F, Ding X, Zhang Z, Bi D, Cheng YT, Chen S, $\mathrm{Li} X$, et al: Regulation of cell death and innate immunity by two receptorlike kinases in Arabidopsis. Cell Host Microbe 2009, 6(1):34-44.

48. Nomura K, Mecey C, Lee YN, Imboden LA, Chang JH, He SY: Effectortriggered immunity blocks pathogen degradation of an immunityassociated vesicle traffic regulator in Arabidopsis. Proc Natl Acad Sci USA 2011, 108(26):10774-10779.

49. Nomura K, Debroy S, Lee YH, Pumplin N, Jones J, He SY: A bacterial virulence protein suppresses host innate immunity to cause plant disease. Science 2006, 313(5784):220-223.

50. Tanaka H, Kitakura S, De Rycke R, De Groodt R, Friml J: Fluorescence imaging-based screen identifies ARF GEF component of early endosomal trafficking. Current biology: CB 2009, 19(5):391-397.

51. Robatzek S: Vesicle trafficking in plant immune responses. Cell Microbiol 2007, 9(1):1-8.

52. Hauck P, Thilmony R, He SY: A Pseudomonas syringae type III effector suppresses cell wall-based extracellular defense in susceptible Arabidopsis plants. Proc Natl Acad Sci USA 2003, 100(14):8577-8582

53. Thilmony R, Underwood W, He SY: Genome-wide transcriptional analysis of the Arabidopsis thaliana interaction with the plant pathogen Pseudomonas syringae pv. tomato DC3000 and the human pathogen Escherichia coli 0157:H7. The Plant journal: for cell and molecular biology 2006, 46(1):34-53.

54. Bartetzko V, Sonnewald S, Vogel F, Hartner K, Stadler R, Hammes UZ, Bornke F: The Xanthomonas campestris pv. vesicatoria type III effector protein XopJ inhibits protein secretion: evidence for interference with cell wall-associated defense responses. Mol Plant Microbe Interact 2009, 22(6):655-664

55. Lee AH, Hurley B, Felsensteiner C, Yea C, Ckurshumova W, Bartetzko V, Wang PW, Quach V, Lewis JD, Liu YC, et al: A bacterial acetyltransferase destroys plant microtubule networks and blocks secretion. PLOS Pathog 2012, 8(2):e1002523.

56. Wang D, Weaver ND, Kesarwani M, Dong X: Induction of protein secretory pathway is required for systemic acquired resistance. Science 2005, 308(5724):1036-1040.

57. Zipfel C, Robatzek S, Navarro L, Oakeley EJ, Jones JD, Felix G, Boller T: Bacterial disease resistance in Arabidopsis through flagellin perception. Nature 2004, 428(6984):764-767.

58. Pecenkova T, Hala M, Kulich I, Kocourkova D, Drdova E, Fendrych M, Toupalova H, Zarsky V: The role for the exocyst complex subunits Exo70B2 and Exo70H1 in the plant-pathogen interaction. J Exp Bot 2011 62(6):2107-2116

\section{Submit your next manuscript to BioMed Central and take full advantage of:}

- Convenient online submission

- Thorough peer review

- No space constraints or color figure charges

- Immediate publication on acceptance

- Inclusion in PubMed, CAS, Scopus and Google Scholar

- Research which is freely available for redistribution

Submit your manuscript at www.biomedcentral.com/submit
() Biomed Central 